\title{
Les relations entre acteurs, les tendances technologiques et les droits des individus
}

\section{Elspeth Guild et Didier Bigo}

\section{(2) OpenEdition \\ 1 Journals}

Édition électronique

URL : http://journals.openedition.org/conflits/939

DOI : $10.4000 /$ conflits.939

ISSN : $1777-5345$

\section{Éditeur :}

CCLS - Centre d'études sur les conflits lilberté et sécurité, L'Harmattan

\section{Édition imprimée}

Date de publication : 1 mars 2003

ISBN : 2-7475-4868-8

ISSN : 1157-996X

\section{Référence électronique}

Elspeth Guild et Didier Bigo, « Les relations entre acteurs, les tendances technologiques et les droits des individus », Cultures \& Conflits [En ligne], 49 | printemps 2003, mis en ligne le 29 septembre 2003, consulté le 30 mars 2021. URL : http://journals.openedition.org/conflits/939 ; DOI : https://doi.org/ $10.4000 /$ conflits.939

Ce document a été généré automatiquement le 30 mars 2021.

Creative Commons License 


\title{
Les relations entre acteurs, les tendances technologiques et les droits des individus
}

\author{
Elspeth Guild et Didier Bigo
}

Les relations entre les Etats et les individus

Pendant longtemps, la théorie de la souveraineté a permis aux gouvernants de tracer une frontière étanche entre les règles internes et externes, 1 ' ordre social et l'anarchie internationale, la police et le social d'un côté, la guerre et le diplomatique de l'autre. D'un côté dans l'Etat weberien règne la rationalité, le choix optimal pour l'individu et l'horizon de la démocratie, de l'autre dans l'Etat westphalien, règne le risque de destruction, le calcul des Etats et l'horizon du conflit. Il existe un « Nous » et un « Eux » clairement désignés, un étranger et un national. Les théories réalistes ont toujours survalorisé ces distinctions et les ont parfois essentialisées en confondant altérité et inimitié.

Les thèses libérales ont au contraire relativisé ces visions de la frontière comme coupure et ont mis en avant le rôle de passage des frontières, de point de jonction positif. Les discours économiques ont aussi, dans la veine libérale, relativisé la prétention de l'Etat au monopole sur le droit de circulation des individus et sur leurs entrées et sorties du territoire national. La notion de frontière s'est détachée du territoire au sens où elle n'en est plus la borne, la limite, l'enveloppe, le lieu de contrôle délimitant l'interne et l'externe. L'européanisation associée à la mondialisation a changé les procédures faisant de la frontière physique d'un Etat une institution. Ces procédures ont alors créé des disjonctions entre frontière ligne, frontière zone et lieux de contrôle. Il s'est construit une dialectique entre liberté de mouvement et protection des frontières qui ne se réduit pas au paradoxe libéral classique de la «back door », de la porte ouverte au sein de la frontière fermée, de la relation entre économie privée ayant besoin de main d'ouvre et sécurité se méfiant des étrangers.

Cette dialectique a bien plus débouché sur un impératif normatif de circulation et de mise en mouvement. Elle s'est structurée comme «dromocratie », où les puissants sont 
ceux qui règnent sur la vitesse, contrôlent celle des autres et déqualifient socialement ceux qui restent sur place ${ }^{1}$. Dans ce dispositif, il devient de plus en plus illégitime de ne pas bouger ou faire bouger, du moins lorsqu'on est riche et consommateur. En revanche, l'impératif de circulation qui vise à accélérer les flux crée de lui-même des lieux de ralentissement et de stockage de plus en plus nombreux où se retrouvent ceux qui n'ont pas les moyens de bouger ou qui ne peuvent le faire sans l'aide des autres : demandeurs d'asile, individus trop pauvres pour quitter les quartiers de relégation... ${ }^{2}$ En interne, on tend à les chasser de la vue et des espaces publics mais en maintenant les espaces de stockage. En externe, on tend à les bloquer en amont ou, s'ils sont partis malgré tout, à orienter leurs "flux » vers des « nasses ", où ils se retrouveront dans des camps comme Sangatte, prêts à être rapatriés. Des deux côtés, en interne comme en externe, les individus pauvres, désaffiliés deviennent suspects lorsqu'ils veulent eux aussi profiter de la circulation sous forme de tourisme et de consommation à leur niveau, mais il est impossible de le dire ouvertement, d'où les jeux de masque successifs.

L'originalité de l'Union Européenne aura été sans doute, à l'époque contemporaine, de consacrer ce fait de circuler et circuler vite comme un "droit " pouvant déboucher sur des sanctions contre l'Etat via les cours de justice européenne. Et, si la facilité de circulation en termes pratiques n'a pas attendu l'européanisation, celle-ci l'a consacrée comme une liberté importante pour tous, à la différence par exemple des accords de l'ALENA en Amérique du Nord. La dialectique liberté contrôle en a été infléchie dans l'espace européen (Schengen comme le Royaume-Uni et l' Irlande) avec la mise en place de dispositifs permettant de faire circuler tout le monde, y compris les étrangers insérés dans l'espace (mais avec le maintien d'une frontière entre cet espace anglocelte et l'espace schengen). Ces dispositifs auraient pu, unifiés, jouer leur rôle, mais, loin de se déployer pleinement, ils ont été assortis de régulations contraires visant à plus et mieux contrôler, à affiner et sérier le contrôle bien plus qu'à l' abolir. C'est au cour de cette dynamique que le réseau Schengen s'est institué et que l'on a voulu harmoniser les politiques de visa à l'égard des pays tiers, tout en maintenant des logiques de souveraineté via l' attribution des passeports et de la vignette visa aux individus. Ceci s'est cristallisé dans des oppositions, non pas nationales entre les pays, mais transnationales en fonction des ministères. Les ministères de la Justice et de l'Intérieur ont essayé de faire prévaloir les "impératifs » de sécurité alors que les ministères des Affaires étrangères et des finances ainsi que les juges du siège ont joué de la primauté d'une diplomatie globale et des règles de droit pour minimiser dans certains cas ces impératifs. Les quinze ans de négociation autour de Schengen montrent le chemin parcouru en ce sens et les jeux chaotiques entre une tendance qui veut toujours plus d'identification des étrangers pour les surveiller et les contrôler et une tendance qui insiste sur l'impératif normatif de la circulation. Les chefs de gouvernements ont souvent appuyé leurs ministères de l'Intérieur et ont réactivé des discours de contrôle d'autant plus sévères que l'adversaire communiste disparu, on pouvait momentanément minimiser le discours sur la liberté de circulation comme facteur de différentiation des régimes politiques entre dictatures et démocraties. Cela a fortement affaibli la portée du droit du demandeur d'asile qui, au lieu d' avoir le droit à être protégé c'est-à-dire abrité (tegere en latin), a été protégé certes, mais au sens militaire de surveiller et d'enclore (praesidere) ${ }^{3}$. Si les chefs de gouvernement ont agi ainsi c'est en fonction aussi de leurs propres peurs, de leurs propres incertitudes sur l'avenir. Le climat d'inquiétude né dans les années 1992-1993 à propos des flux massifs 
de migrants arrivant de l'Est (Russie) ou du Sud (Algérie) a été transformé en problème politique par les hommes politiques qui ont fait de la gestion des sentiments d'insécurité une part de leur activité routinière alors que jusque-là ils ne se saisissaient du problème qu'exceptionnellement. Cette conjoncture a duré et pris le pas sur celle de la confiance retrouvée dans les progrès de la démocratie libérale et, malgré des fluctuations récentes, elle sert de marqueur dans le jeu politique et permet des conversions de la gauche vers la droite en particulier.

C'est dans ce contexte qu'il faut analyser la régulation des visas et la gestion de la police à distance par les professionnels de la sécurité. L' individu n'a plus été une valeur porteuse en soi, il a été perçu comme un collectif, comme un flux déstabilisant et il a été souvent déshumanisé avec les terminologies de masse, de foule ou même de flux. La gestion des passeports et des visas s'en est ressentie elle aussi. L'attribution du visa ne « juge » presque plus l'individu mais son appartenance à une catégorie prédéfinie.

Les relations des Etats à la Commission européenne et de celle-ci aux individus

Le visa Schengen a encore plus complexifié les relations entre Etat et individu. L'Etat n'a plus véritablement le monopole, même de principe, sur la circulation des individus. La construction européenne, en posant la libre circulation des personnes comme principe a perturbé le fonctionnement monopolistique. Les Etats ont dû commencer à négocier entre eux pour maintenir un contrôle qu'ils exerceraient collectivement à l'égard de tous leurs citoyens et des étrangers vivant dans l'espace européen. Le clivage territorial induit par le Royaume-Uni et l'Irlande a fait sauter les adéquations simples entre territoire européen et espace des accords intergouvernementaux sur la circulation des personnes et leurs mesures compensatoires. Les masques successifs pris par le «laboratoire Schengen", puis par «l'acquis Schengen", de même que l' invention laborieuse des piliers dans Maastricht puis leur redéfinition dans Amsterdam, n'ont en rien créé les conditions d'une clarification. La Commission, via la politique des visas sur les passeports a essayé de gérer cette situation en essayant de poser le principe de la libre circulation avec les pays amis (dont les ressortissants ne sont pas soumis à visa) en contrepartie d'une vérification des identités des personnes désirant voyager dans l'Union dès leur point de départ et la mise en place de stratégies de mise à l'écart des étrangers indésirables. A l'intérieur même de la Commission, selon que l'on appartienne au premier pilier titre IV ou au troisième pilier, les contradictions entre libre circulation et renforcement des mesures coercitives à l'égard des touristes pauvres ont pris un tour exacerbé, dupliquant les oppositions idéologiques entre partis et entre gouvernements. Les discours sur la confiance réciproque, sur les mesures harmonisées ont caché la prolongation et l'exacerbation des luttes pour maintenir des marges de manouvre intactes malgré le nouveau cadre. Les pratiques d'exception, de méfiance à l'égard des autres parties contractantes ont empêché une harmonisation, et lorsque celle-ci a eu un semblant d'effet, c'était pour un renforcement de solutions répressives et peu démocratiques. La tentation d'utiliser la politique des visas pour éviter les critiques des ONG autour des zones d'attente et des frontières a été renforcée. La construction d'une stratégie de police à distance a conduit au renforcement de la suspicion à l'égard des étrangers en la teintant de racisme car la distribution des pays soumis ou non à visa tend par trop à ressembler aux stéréotypes même des Européens sur les races inférieures et à leur imaginaire colonial. Le critère de la pauvreté a été opposé à celui de la libre circulation au nom du risque migratoire en empêchant les plus pauvres de voyager ou en encadrant de manière bien plus restrictive leurs mouvements, surtout si on le compare avec les plus riches. On a vu les Etats, non plus 
s'intéresser au régime politique de l'autre Etat mais au comportement de certaines minorités de ce pays ou au risque de nomadisme volontaire ou forcé des individus vivant sur place.

En ce sens les rapports Etat, Commission, individu se sont aussi modifiés car certains individus ont été promus comme ennemis non désignés des Etats à la place ou en accompagnement des anciens régimes hostiles. L' européanisation des pratiques de contrôle concernant les visas a mis alors en lumière les décalages entre qui nous sommes, quelles sont nos valeurs et qui suspectons nous de ne pas les respecter. On a changé les technologies de contrôle en abandonnant la stricte sécurisation à la frontière territoriale pour une sécurisation en amont de certains flux de personnes considérées comme dangereuses. La mise en place plus systématique de profils à risque regroupant les individus en catégories à risque a permis de sortir de la pure logique judiciaire individuelle à qui l'on reproche sa lenteur et de la logique de la stricte appartenance étatique d'un individu qui est trop globale. Entre l'Etat et l'individu on s'est essayé à utiliser les catégories de minorités, d'ethnies, de lieux dangereux, etc. en empreintant un certain discours, voire un certain savoir aux sociologues et aux criminologues. La police à distance dans l'espace s'est couplée avec une police à distance dans le temps où les profils de risque sont censés anticiper les actions des individus ${ }^{4}$. Mais les systèmes de justification sont encore suffisamment récents pour laisser apparaitre leur part d' arbitraire fondateur. Les résistances des individus sont multiples et passent par la recherche d'appui au sein des institutions en charge des libertés ou à travers des procédures détournant les contrôles. Cela crée une logique en spirale où les procédures d'identification veulent s'émanciper du bon vouloir de l'individu et objectiver totalement ce dernier, le réduire à n'être qu'un corps et non plus une personne pouvant jouer avec ou se jouer des administrations. L'hyper technologisation est alors présentée comme une solution pour redonner aux Etats ou à la Commission et aux Etats le dernier mot sur la circulation des " personnes".

La technologisation comme ultra-solution

Le 11 septembre 2001 a été l'occasion d'une réactivation extraordinaire de cette tendance à la technologisation des identités et à l'appel à des procédures d'identification biométrique que l'on espère indépendantes des capacités de dire et de faire des personnes ${ }^{5}$. On a vu un discours d'abord nord américain sacraliser à nouveau la frontière et sa défense. L'idée d'un bouclier électronique arrêtant les attentats a pris la suite de celle d'un bouclier arrêtant les missiles nucléaires. Mais, très vite, le Homeland Security Department a fait savoir qu'il considérait que la sécurité des américains se jouait à l'extérieur des frontières, et non lorsque ces dernières sont franchies ${ }^{6}$. Il a certes renforcé le national Security Entry Exit Registration System en exigeant des entretiens renforcés avec un groupe de nationalités qui ressemble comme un jumeau à la liste de l'annexe $5 \mathrm{~b}$ de Schengen mais il a surtout mis au cour du dispositif une réflexion sur les passeports et les visas en prônant une technologisation accrue de ces derniers ${ }^{7}$. Il a même essayé de contrer la mainmise des départements d'Etat et de la défense sur la politique extérieure, en particulier avec les alliés britanniques. Les soutiens à la politique du Homeland Security Department et aux réformes de l'INS sont venus très largement de certaines sociétés privées qui avaient déjà fait des recherches sur les données biométriques mais ne trouvaient pas de marché pour leurs produits. 
De nombreuses entreprises ont saisi l'opportunité du 11 septembre. Pour ne citer qu'un exemple la société ZN Vision technologie a ainsi vanté son système de reconnaissance faciale automatique permettant, selon ses promoteurs, de vérifier l'identité du détenteur du document en comparant ses traits et ceux de la photo du document présenté, dans une lettre datée du 18 octobre $2001^{8}$. Ces entreprises ont mis l'accent sur leur capacité à dire la véritable identité physique de la personne, indépendamment des papiers présentés par cette dernière. Il suffirait selon elles d'investir dans des technologies plus lourdes, où les gouvernements centraux des pays développés, par l'instauration de bases de données biométriques reliées entre elles via l'électronique ou via une carte dont $\mathrm{l}^{\prime}$ individu serait porteur, pourraient coopérer sans que ce même individu ne puisse intervenir activement et "altérer", à son avantage cette coopération. L'argumentaire de ces diverses présentations est de faire passer l'idée de contrôles biométriques où l'individu n'est même plus conscient d'être contrôlé, en insistant sur le fait que, dans les contrôles via la reconnaissance faciale et celle des iris, une fois que les données ont été enregistrées, les individus ont simplement à passer sous un portique sans avoir à s'arrêter ou à être touchés'.

Le salon Milipol qui se tient alternativement au Qatar et à Paris s'est fait une spécialité mondiale d'inviter ces sociétés spécialisées dans toute l' industrie et les services qui peuvent être utiles aux polices dans le monde. De fait, on a vu ces quatre dernières années une montée en puissance considérable des sociétés proposant ces types de savoir-faire, souvent à la suite de la reconversion de programmes commencés lors de l'initiative de défense stratégique de Ronald Reagan et qui, à court d'ennemi soviétique, $s^{\prime}$ étaient rabattues sur la surveillance plus individualisée des personnes passant les frontières et sur la biométrie ainsi que l'électronique de précision.

Ce discours commercial sur la solution au terrorisme comme à l'immigration clandestine ou à la détection de faux documents grâce aux techniques biométriques a été repris in extenso par certains hommes politiques et certains ministères de l'Intérieur de l'Union Européenne, à l'unisson avec le Homeland Department. Si l'on a beaucoup évoqué les données biométriques liées à l'ADN et les empreintes digitales que l'on stockerait sur des "puces" électroniques, des chip cards, il semble que ces systèmes soient néanmoins onéreux et qu'ils soient momentanément délaissés au profit de technologies ne demandant qu'un effort initial limité de la personne pour les prélèvements nécessaires (reconnaissance de l'iris ou reconnaissance faciale). Les systèmes plus lourds des empreintes et de l'ADN seraient alors réservés à des populations que l'on a pu stocker dans des camps ou emprisonnés, et que l'on croit pouvoir immobiliser plus longtemps à des fins de contrôle alors que les autres techniques seraient utilisées pour la surveillance d'une population plus globale qui les accepteraient plus facilement. Les personnes dans les camps et centres de rétention seraient soumises aux technologies des empreintes digitales comme avec la mise en place du système Eurodac ${ }^{10}$ qui s 'est effectuée officiellement le 15 janvier 2003 mais qui avait débuté par des relevés d'empreintes plus d'un an avant sur les demandeurs d'asile et certaines catégories d'immigrants illégaux retenus en centre. Les millions de personnes transitant par les aéroports, les aérogares, ou même des réseaux autoroutiers ou une ville comme à Londres depuis la "Congestion charge ", seraient, elles, sous surveillance vidéo et reconnaissances des iris ou de la face par des logiciels comparant en quelques secondes la caméra avec des profils suspects et lançant un «warning » d'interception. Mais face aux aléas techniques de réglages de la sensibilité de ces programmes entre temps d'attente et fiabilité, il semble que l'on s'oriente sur 
des technologies encore plus simples, dépendant certes en partie des documents papiers et de la bonne volonté du porteur de les présenter, mais qui prendraient la mesure des corps et apporteraient une vérité que l'on veut croire indépendante de l'individu. Ainsi, on intégrerait très bientôt au passeport ou à la vignette visa une photographie d'identité électronique permettant une fois reliée à un logiciel de lecture des points clés du visage de lire la structure du visage et de la comparer éventuellement avec le même logiciel couplé à une caméra filmant l'individu présentant le document. Dans ce cas, théoriquement, même entre frères, le logiciel permet de faire la différence, et est capable de certifier que la personne est bien celle dont la photo a été prise sur les documents. Les Américains exigeraient aussi que dès maintenant tous les Etats délivrant des passeports soient astreints à le faire selon des normes techniques identiques permettant leur lecture optique et une photographie électronique et ils menacent de supprimer les visas waiver programme à tous les pays qui refuseraient de $s^{\prime}$ aligner sur eux ${ }^{11}$. Au delà d'une science fiction des technologies biométriques qui se rapprochent néanmoins à grand pas et que propagent les médias, c'est plutôt autour de ces derniers procédés que les recherches opérationnelles s'orientent et donc c'est à travers un renforcement des techniques de passeports et de visas (mais surtout de ce dernier dans l' Union) que l'on croit pouvoir trouver des «solutions $»^{12}$. Cet avenir du visa par la haute technologie va sans doute compliquer les contrefaçons, permettre de lier l' amont et l'aval des contrôles, mais cette identification à l'échelle européenne ou/ et transatlantique visant à un bertillonage planétaire réactivant l'anthropométrie cette fois digitalisée ne règle en rien les procédures de sélection des personnes et des groupes à qui on va $\mathrm{l}^{\prime}$ appliquer ${ }^{13}$. La technologie ne résoudra pas les contradictions politiques ${ }^{14}$.

Droits et résistances des individus

L'analyse des pratiques d'obtention du visa et des logiques de contrôles qui les accompagnent, montre que nous sommes loin de l'harmonisation annoncée par les textes. Certes, beaucoup de personnes voyagent dans l'Union et l'espace Schengen. D'abord ceux qui n'ont pas besoin de visa. Ensuite ceux qui l'obtiennent néanmoins. Mais pour ces derniers les frustrations sont fortes. Ce sont ces petites tragédies du quotidien qui montrent le mépris des Européens pour les autres qui risquent de détériorer la situation internationale. Obtenir un visa après avoir été soupçonné à tort crée un sentiment d'injustice, même si on l'a finalement eu à temps. La focalisation sur les individus à risque et non plus sur l'appartenance d'un individu à un régime politique d'un Etat a fondamentalement complexifié la relation au visa. Elle a généralisé la vieille notion d'indésirable et l'a réactualisé sans pudeur et en oubliant son passé venant de l'empire Austro-hongrois. Le terme est devenu commun dans le langage des fonctionnaires de tout l'espace Schengen et ne choque que quelques Britanniques. Il n'y a même pas eu unité autour de cette catégorie des indésirables et phénomène de folkdevil. Les « indésirables » dans un pays ne le sont pas forcément dans un autre, et la création d'une bourse d'échange des peurs européennes à travers les systèmes techniques de surveillance et des comités plus ou moins occultes, ne règle pas le problème sur le fond.

Si la légitimité de refuser l'entrée sur le territoire à des criminels est forte, elle s'affaiblit sérieusement lorsque l'on évoque la gestion des flux migratoires et devient évanescente lorsque l'on refuse à quelqu'un, une première entrée sur le territoire en fonction de son appartenance à un profil de groupe à risque migratoire. Privés des 
individus pauvres de retrouver leur famille sur le territoire européen ou de simplement venir le visiter ne se justifie pas.

Les techniques de découragement d'obtention du visa pour les individus les plus pauvres ou ceux qui appartiennent à certains groupes ethniques ne vont pas dans le sens de l'ouverture européenne, en particulier lorsque cela s' applique à des pays qui ont vocation à rejoindre l'Union ou à être dans le cercle des « amis ".

Le droit à la libre circulation - au voyage, au tourisme - doit être la priorité, et les préoccupations de sécurité, l'exception. Or, dans certains pays européens - mais pas tous -, il y a une tendance à l'inversion de ces priorités et une exagération des "dangers", des "risques» qui viendraient de l'étranger par le jeu des classes politiques et de la saisie de la question du sentiment d'insécurité comme enjeu politique. Un climat d'inquiétude, de malaise social est responsable d'attitudes générales peu favorable aux étrangers venant du tiers monde et marqués par leur couleur de peau, encore faut-il ne pas entretenir ce climat, mais le combattre, en refusant la fabrication de stéréotypes par des marchands de peur qui ont tendance ensuite à en vivre.

Les hommes politiques comme les services de l'Etat doivent infléchir ces discours de peurs, au lieu de les suivre sous prétexte qu'ils seraient le reflet de l'opinion. Il faut insister sur la nécessité de relancer un discours d'ouverture et d'espoir sur les capacités d'intégration de l'Union et sur les avantages de la circulation généralisée des hommes qui n'a nullement débouché sur l'invasion des pays riches par les plus pauvres comme le montre les derniers élargissements. Comme nous l'avons signalé à plusieurs reprises, les pauvres grecs n'ont nullement submergés l'Allemagne ou la France avec l'entrée de celle-ci dans l'Union.

On ne peut continuer à élaborer des politiques sur la base de catégories aussi ambiguës que celle d'indésirables, non qu'il n'y ait pas à se protéger de certains individus criminels, mais parce que l'extension au-delà du crime des préoccupations de sécurité intérieure ne peut guère se faire en commun et a une légitimité discutable.

De même la « police à distance » qui tend à contrôler les personnes loin du territoire où ils peuvent avoir des recours si leurs droits sont mis en danger, tend à être cynique - si tel est l'objectif - ou maladroite. Il ne peut être question de transnationaliser les contrôles tout en circonscrivant territorialement les droits de recours. Les juges comme les citoyens actifs des démocraties ne le toléreront pas.

\section{NOTES}

1. . Sur la notion de « dromocratie », Virilio Paul, Vitesse et politique, Paris, Editions galilée, 1977.

2. . Voir les travaux de Zygmunt Bauman et les réflexions de Gérard Noiriel qui précisait : on n'a jamais autant enfermé les individus que depuis que l'on a proclamé la libre circulation en faisant l'historique depuis la révolution française de ce qui n'est qu'un paradoxe apparent. 
3. . Je remercie Emmanuel-Pierre Guittet de m'avoir fourni ces précisions sémantiques sur la protection. Sur la notion de protection voir Didier Bigo (et al.), La fonction de protection, op. cit.

4. . Sur ce point et le parallèle avec l' ouvrage de Philip K. Dick « Minority Report » voir Bigo Didier, « La recherche proactive et la gestion du risque », Déviance et société, 1997, vol 21 4, pp. 423-429. Voir aussi « globalisation of (in)security », Traces, op. cit.

5. . Et cela rappelle ce qui s' était passé après l'attentat d'Orsini contre Napoléon III avec le développement des techniques d'identification qui lui fut subséquent. Voir là encore les travaux de Gérard Noiriel.

6. . Voir les travaux de Philippe Bonditti : L' antiterrorisme aux Etats-Unis. De la menace soviétique aux Rogue States. La construction de l'ennemi terroriste depuis 1980, mémoire DEA IEP, Paris, septembre 2001 ; Les organisations de lutte antiterroriste aux Etats-Unis, rapport DAS, 2002.

7. . Voir « The Enhanced Border Security and Visa Reform Act 2002 » (EBSVRA) et la technologie dite Lincoln visa qui comporte des informations biométriques digitalisées. Powell C Testimony before the house select committee on homeland security 11/07/2002.

8. . Voir en annexe du second volume, le document de la société ZN vision : « Reconnaissance faciale automatique pour la sécurité du transport aérien ».

9. Certains argumentaires expliquant cyniquement que ce n'est pas le contrôle qui gêne les personnes mais la conscience du contrôle.

10. . Un CD-Rom publicitaire d'Eurodac a même été distribué pour justifier ces technologies au nom de la libre circulation et des vrais demandeurs d'asile. Un exemplaire du CD Rom se trouve au Centre d' Etudes sur les Conflits. Sur la justification de l'injustifiable, voir les travaux théoriques de Martin Heisler, ISA Chicago 2002, à paraître.

11. Sur la polémique créée autour de l'accord donné par la Commission aux Etats-Unis d'avoir des documents permettant le contrôle de l'identité des voyageurs avant leur arrivée sur le territoire américain et sur ce projet de passeport niant le droit des autres Etats à avoir leurs propres règles (et donc leur souveraineté sur " leurs » individus) voir les travaux de Mark Salter, Rights of passage : the passport in international relations, Boulder, Lynne Rienner, 2003. Voir également les travaux du groupe ELISE : http:// www.eliseconsortium.org ; et ceux de Statewatch http://www.statewatch.org 12. . Pour une approche critique Wright Steve, " An appraisal of technologies of political control », Omega foundation, European parliament, Scientific and technological options assessment, 06/01/1998 (à la base du rapport critique sur Echelon et le plan UE-FBI).

13. . Le projet de du VIS (système d'information visa) qui se met en place actuellement semble aller dans ce sens. Nous y reviendrons dans un autre article.

14. Sur cette idée d'une identification transatlantique comme un au-delà des identifications nationales, il faut relier les recherches des historiens et des politistes travaillant sur la surveillance. Voir à ce sujet le colloque organisé par le CERI et le Centre d'Etudes sur les Conflits sous la direction de Nathalie Bayon les 24 et 25 mars 2003. Les travaux de Gérard Noiriel à propos de la tyrannie du national et de $\mathrm{l}^{\prime}$ identification nationale devraient être mis en parallèle avec les tendances contemporaines que nous analysons. 
INDEX

Mots-clés : droits de l'Homme, migration, bases de données 